\title{
Rational Design of Hit Compounds Targeting Staphylococcus aureus Threonyl-tRNA Synthetase
}

\author{
Mariia Yu. Rybak, Olga I. Gudzera, Oksana B. Gorbatiuk, Mariia O. Usenko, Sergiy M. Yarmoluk,
} Michael A. Tukalo, and Galyna P. Volynets*

Cite This: ACS Omega 2021, 6, 24910-24918

Read Online<smiles>O=[N+]([O-])c1ccc(-c2csc(N/N=C/c3cc(Br)cc(Br)c3O)n2)cc1</smiles>

\section{Staphylococcus aureus ThrRS IC ${ }_{50}=56.5 \mu \mathrm{M}$}

ABSTRACT: Staphylococcus aureus is one of the most dangerous nosocomial pathogens which cause a wide variety of hospitalacquired infectious diseases. $S$. aureus is considered as a superbug due to the development of multidrug resistance to all current therapeutic regimens. Therefore, the discovery of antibiotics with novel mechanisms of action to combat staphylococcal infections is of high priority for modern medicinal chemistry. Nowadays, aminoacyl-tRNA synthetases are considered as promising molecular targets for antibiotic development. In the present study, we used for the first time S. aureus threonyl-tRNA synthetase (ThrRS) as a molecular target. Recombinant $S$. aureus ThrRS was obtained in the soluble form in a sufficient amount for inhibitor screening assay. Using the molecular docking approach, we selected 180 compounds for investigation of inhibitory activity toward ThrRS. Among the tested compounds, we identified five inhibitors from different chemical classes decreasing the activity of ThrRS by more than $70 \%$ at a concentration of $100 \mu \mathrm{M}$. The most active compound 2,4-dibromo-6-\{[4-(4-nitro-phenyl)-thiazol-2-yl]-hydrazonomethyl\}-phenol has an $\mathrm{IC}_{50}$ value of $56.5 \pm 3.5 \mu \mathrm{M}$. These compounds are not cytotoxic toward eukaryotic cells HEK293 $\left(\mathrm{EC}_{50}>100 \mu \mathrm{M}\right)$ and can be useful for further optimization and biological research.

\section{INTRODUCTION}

Staphylococcus aureus is an opportunistic Gram-positive pathogen that causes a wide range of hospital-acquired human diseases ranging from skin infections and abscesses to much more severe endocarditis, osteomyelitis, pneumonia, meningitis, sepsis, and so forth. ${ }^{1-8}$ The major problem of staphylococcal infection treatment is the multidrug resistance to all antibiotics currently used in clinic, including methicillin, vancomycin, daptomycin, and linezolid. ${ }^{9-15}$ Therefore, the development of antistaphylococcal agents with novel mechanisms of action is of urgent need.

Nowadays, aminoacyl-tRNA synthetases are recognized as promising molecular targets for antibiotic development. ${ }^{16-18}$ Aminoacyl-tRNA synthetases are key enzymes in protein synthesis which ligate amino acids to cognate transfer RNAs being involved in the early stages of translation of the genetic code. Most living cells possess 20 aminoacyl-tRNA synthetases for each of the standard amino acids. These enzymes are divided into two classes, class I and class II, which have different folds of catalytic domain and different preferences for the hydroxyl group of the tRNA. ${ }^{19,20}$ Aminoacyl-tRNA synthetases possess some structural divergence in prokaryotic and eukaryotic organisms which increases the possibility for development of selective inhibitors toward enzymes from pathogenic microorganisms in comparison with human homologues. Aminoacyl-tRNA synthetases are conservative among bacteria, suggesting that compounds targeting these enzymes may demonstrate a wide range of antibacterial activity. A number of available crystal structures of aminoacyl-tRNA synthetases provide a basis for receptor-based drug design and virtual screening. Aminoacyl-tRNA synthetases are well expressed in the soluble form and can be used for highthroughput screening. Threonyl-tRNA synthetase (ThrRS) is a promising molecular target for malaria treatment. ${ }^{21,22}$ For

Received: July 16, 2021

Published: September 16, 2021 


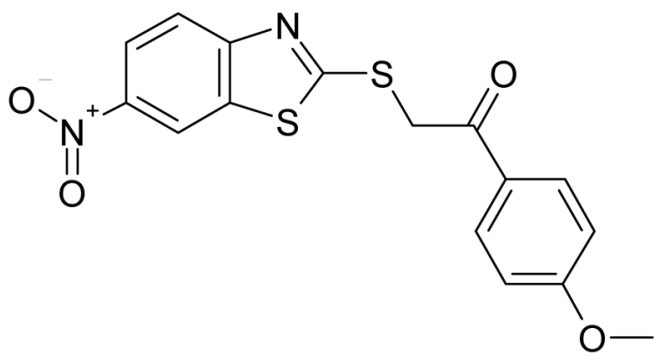

a

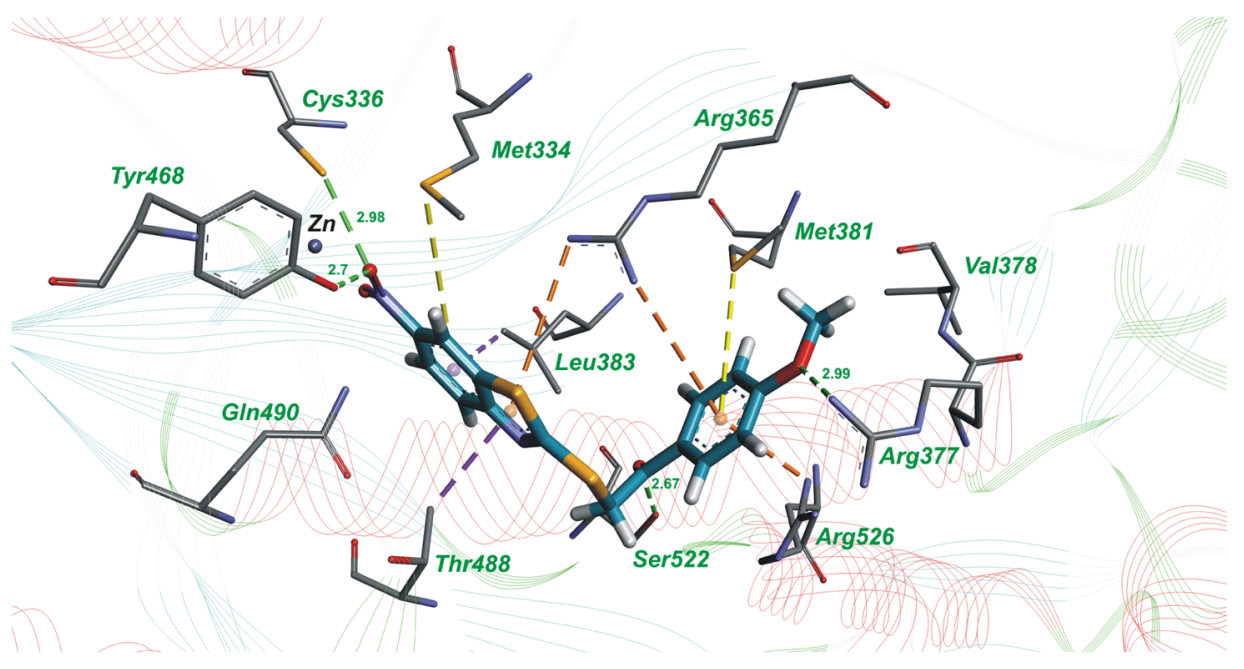

b

Figure 1. Chemical structure of 1-(4-methoxy-phenyl)-2-(6-nitro-benzothiazol-2-ylsulfanyl)-ethanone (compound 1) (a). The complex of compound 1 with amino acid residues in the active site of $S$. aureus ThrRS. The hydrogen bonds are shown by green dashed lines with the distances indicated in $\AA$, the hydrophobic interactions are presented by magenta dashed lines, $\pi$-sulfur interactions are shown by yellow dashed lines, and $\pi$ cation interactions are indicated with orange dashed lines (b).

example, borrelidin, polyketide macrolide, isolated from Streptomyces species, is a potent inhibitor of ThrRS from Plasmodium falciparum. ${ }^{23,24}$ Due to high toxicity of this compound, a series of borrelidin derivatives with lower toxicity has been synthesized. ${ }^{22,25,26}$ The inhibitors of ThrRS from Haemophilus influenza, Escherichia coli, and Burkholderia thailandensis possessing antibacterial activity have been reported among sulfonamide derivatives. ${ }^{27}$ Recently, the inhibitors of Salmonella enterica ThrRS among quinazolinone derivatives have been identified using fragment-based target hopping assay by Guo et al. ${ }^{28}$ The most active compound demonstrates an $\mathrm{IC}_{50}$ value of $1.4 \mu \mathrm{M}$ toward Salmonella enterica ThrRS. It should be noted that this compound revealed antibacterial activity toward Escherichia coli ATCC25922 and S. enterica with an MIC value of $16 \mu \mathrm{g} / \mathrm{mL}$ and toward S. aureus ATCC29213, S. aureus R3708, and Enterococcus faecalis ATCC29212 with an MIC value of $32 \mu \mathrm{g} /$ $\mathrm{mL} .^{28}$ Furthermore, these authors performed structure-based optimization of quinazolinone-threonine hybrids and discovered the inhibitor of $S$. enterica $\mathrm{ThrRS}$ with an $\mathrm{IC}_{50}$ value of 0.5 $\mu \mathrm{M}$ and MIC values of $16-32 \mu \mathrm{g} / \mathrm{mL}$ toward the tested bacterial strains. $^{29}$

For the best of our knowledge, none of the small-molecular inhibitors of ThrRS from S. aureus have been reported in scientific literature so far. Therefore, the aim of this study is to obtain recombinant $S$. aureus ThrRS and identify inhibitors of this enzyme with molecular docking into the available crystal structure of ThrRS. ${ }^{30}$

\section{RESULTS AND DISCUSSION}

Cloning and Purification of $S$. aureus Threonyl-tRNA Synthetase. The gene encoding S. aureus ThrRS (1938 bp) with restriction sites for NcoI and HindIII was synthesized by ATG/biosynthetics GmbH (Merzhausen, Germany). To obtain the required amount of the $S$. aureus ThrRS gene for ligation, we performed preparative restriction of $5 \mu \mathrm{g}$ of plasmid DNA pGE-ThrRS(NcoI-HindIII) from the clones of E. coli Top10 cells with NcoI and HindIII. The linear DNA fragment was purified from gel using the NucleoSpin gel extraction kit (Macherey-Nagel) protocol.

The ThrRS gene fragment was ligated with a dephosphorylated vector $\mathrm{pET} 28 \mathrm{~b}$ linearized with $\mathrm{NcoI}$ and HindIII. E. coli cells TOP10 were electroporated with the plasmid pET28bThrRS. Plasmid DNA of clones with the correct fragments was prepared with a GeneJET Plasmid Miniprep Kit (Thermo Scientific). Transformation efficiency was analyzed with NcoI and HindIII. All tested plasmids contained a fragment of the expected size.

The most optimal conditions for expression of recombinant $S$. aureus ThrRS in the soluble fraction are the following: E. coli BL21(DE3)pLysS cells in LB medium, with induction by 0.25 $\mathrm{mM}$ isopropyl $\beta$-D-1-thiogalactopyranoside (IPTG) for $3 \mathrm{~h}$ at 


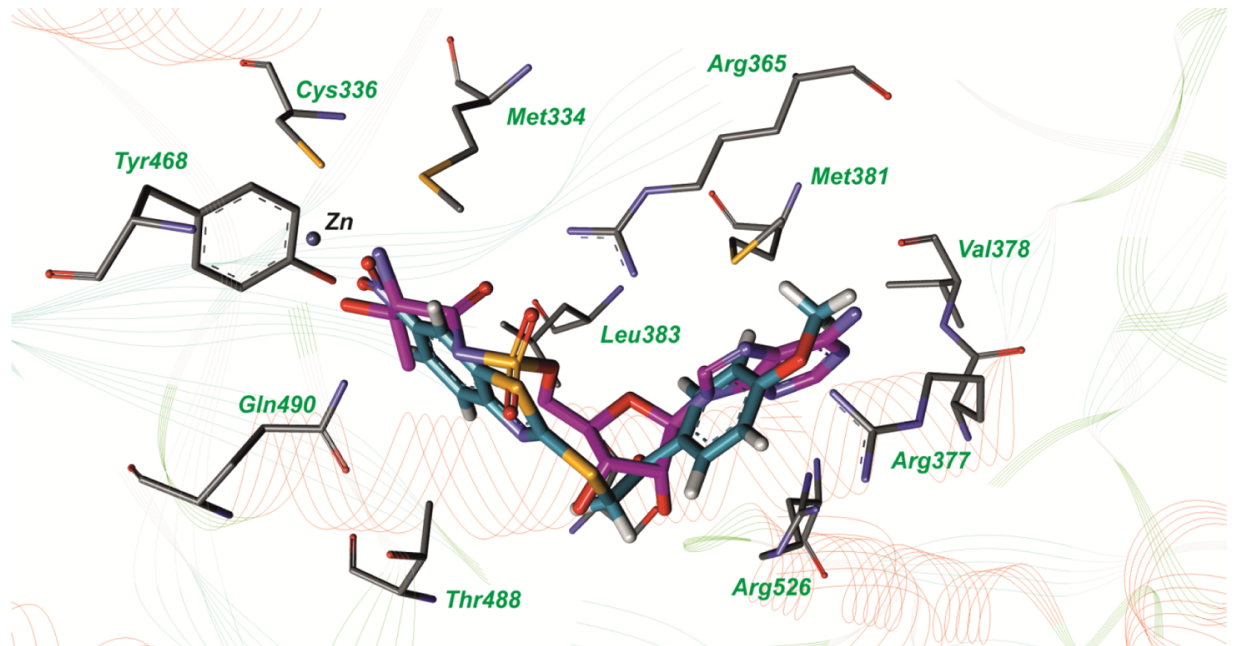

Figure 2. Superposition of the threonyl adenylate analogue, extracted from the crystal structure with the PDB accession code: 1NYQ (carbon atoms are labeled with magenta color) and compound $\mathbf{1}$ (carbon atoms are labeled with blue color) in the active site of S. aureus threonyl-tRNA synthetase.

$37{ }^{\circ} \mathrm{C}$ or E. coli Lemo21(DE3) cells in $2 \mathrm{YT}$ medium supplemented with $250 \mu \mathrm{M}$ L-rhamnose at $30{ }^{\circ} \mathrm{C}$ during 16-18 h. The procedure for selection of optimal expression conditions for $S$. aureus ThrRS is available in Supporting Information (Figures S1-S10).

The protein was purified by DEAE Sepharose anion chromatography using a salt gradient from 25 to $500 \mathrm{mM}$ $\mathrm{NaCl}$ (Figures S11, S12). The resulting eluate was concentrated and loaded onto a pre-equilibrated Heparin Sepharose 6 Fast Flow column and then eluted using the $\mathrm{KCl}$ gradient (from 0 to $300 \mathrm{mM}$ ) (Figure S13). The protein was concentrated to $3.5 \mathrm{mg} / \mathrm{mL}$ and stored with $50 \%$ glycerol at $-80{ }^{\circ} \mathrm{C}$. The total yield of purified ThrRS ( $>95 \%$ purity) is 10 $\mathrm{mg}$ from $1 \mathrm{~L}$ starting bacterial culture. This protocol of purification was used for obtaining the required amount of recombinant $S$. aureus ThrRS for compound screening.

Rational Design of $S$. aureus ThrRS Inhibitors. In order to identify small-molecular inhibitors of $S$. aureus ThrRS, we have performed molecular docking of OTAVA compound library containing 124,831 compounds. ${ }^{31}$ According to molecular docking results, we have selected 180 compounds for investigation of their inhibitory activity toward $S$. aureus ThrRS in an aminoacylation assay. The inhibitory activity for all tested compounds (\%) (measured at least in duplicates) is available in Supporting Information (Table S1). Among the tested compounds, we identified active compounds belonging to five chemical classes-the derivatives of 2-(benzothiazol-2ylsulfanyl)-1-phenyl-ethanone, 2-phenoxy- $N$-phenyl-acetamide, 4-phenyl-2-propionylamino-thiophene-3-carboxylic acid ethyl ester, 1-phenyl-pyrrolidine-2,5-dione, and $N$-benzylidene- $N^{\prime}$ (4-phenyl-thiazol-2-yl)-hydrazine.

The compound 1-(4-methoxy-phenyl)-2-(6-nitro-benzothiazol-2-ylsulfanyl)-ethanone (compound 1) inhibits $S$. aureus ThrRS by $88.47 \%$ at a concentration of $100 \mu \mathrm{M}$. The $\mathrm{IC}_{50}$ value for this compound is $158 \pm 72 \mu \mathrm{M}$ (measured in duplicates). According to molecular docking results, this compound interacts simultaneously with adenine-binding and amino acid-binding regions of ThrRS (Figure 1). The superposition of compound $\mathbf{1}$ and the threonyl adenylate analogue, extracted from the crystal structure of ThrRS (PDB ID: 1NYQ), in the active site is presented in Figure 2. As it can be seen in Figure 1, 4-methoxy-phenyl interacts with amino acid residues in the adenine-binding site and forms a hydrogen bond with Arg377, the benzothiazol ring interacts with the amino acid residues in the threonyl-binding region, and the nitro group at the C6 position of this heterocycle builds hydrogen bonds with Cys336 and Tyr468.

In the present study, we tested six derivatives of 2-phenoxy$N$-phenyl-acetamide for inhibitory activity toward $S$. aureus ThrRS. As it can be noticed from Table 1, the most active compound 2-(2-tert-butyl-phenoxy)- $N$-(2-methyl-5-nitro-phenyl)-acetamide (compound 2) inhibits $S$. aureus ThrRS by $85.59 \%$ at a concentration of $100 \mu \mathrm{M}$. According to molecular docking results, tert-butyl of this compound interacts with the amino acid residues in the adenine-binding region of ThrRS, the nitro-phenyl ring interacts with the amino acid residues in the threonyl-binding region, and the nitro group forms the metal-acceptor bond with $\mathrm{Zn}$ (Figure 3).

We have made superposition of the most active compounds $\mathbf{1}$ and $\mathbf{2}$ in the aminoacyl-adenylate binding site of ThrRS (Figure S14). It was found that these ligands have a similar binding mode. Both compounds 1 and 2 interact simultaneously with adenine- and threonyl-binding regions and form $\pi$-cation interactions with the same residues Arg365 and Arg526. Possibly, the formation of hydrogen bonds of the nitro group in compound $\mathbf{1}$ is more profitable for inhibitory activity than the formation of the metal-acceptor bond of the nitro group in compound $\mathbf{2}$ with $\mathrm{Zn}$.

In this research, three derivatives of 4-phenyl-2-propionylamino-thiophene-3-carboxylic acid ethyl ester were tested for inhibitory activity toward $S$. aureus ThrRS. As it can be seen from Table 2, the most active compound-4-(4-chlorophenyl)-2-[2-(2,6-dimethyl-morpholin-4-yl)-acetylamino]-thiophene-3-carboxylic acid ethyl ester (compound 8), inhibits $S$. aureus ThrRS by $78.33 \%$. According to molecular docking results, 2,6-dimethyl-morpholine heterocycle of this compound interacts with the amino acid residues in the adenine-binding region of ThrRS and forms a hydrogen bond with Arg377 and 4-chloro-phenyl interacts with the threonyl-binding region (Figure 4).

Among the investigated compounds, we have found two inhibitors of $S$. aureus ThrRS belonging to 1-phenyl- 
Table 1. Structures and In Vitro Inhibitory Activity of 2Phenoxy- $\mathrm{N}$-phenyl-acetamide Derivatives toward $\mathrm{S}$. aureus ThrRS

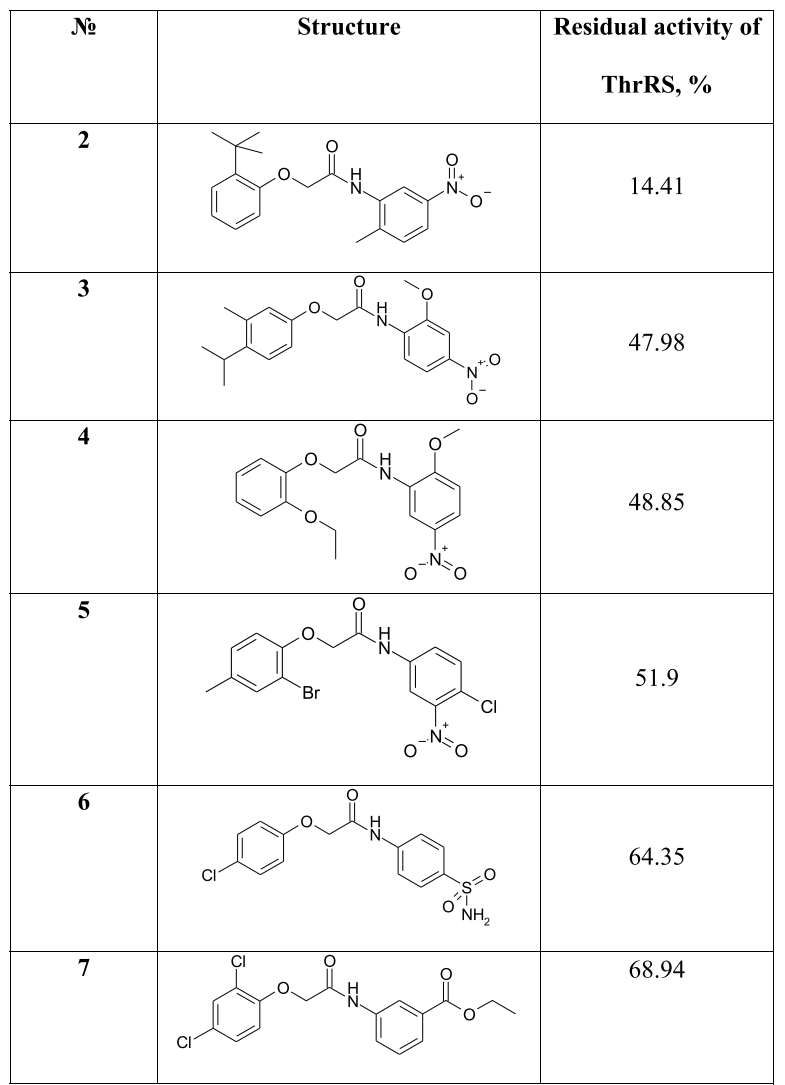

pyrrolidine-2,5-dione derivatives (Table 3). The compound 2[1-(4-iodo-2-methyl-phenyl)-2,5-dioxo-pyrrolidin-3-ylsulfanyl]-nicotinic acid (compound 11) inhibits ThrRS by $78.62 \%$ at a concentration of $100 \mu \mathrm{M}$. According to molecular docking results, the nicotinic acid moiety of this compound interacts with the adenine-binding region of ThrRS and forms a
Table 2. Structures and In Vitro Inhibitory Activity of 4Phenyl-2-propionylamino-thiophene-3-carboxylic Acid Ethyl Ester Derivatives toward S. aureus ThrRS

\begin{tabular}{|l|c|c|c|}
\hline № & Residual activity of \\
\hline ThrRS, \% \\
\hline 10
\end{tabular}

hydrogen bond with Arg377 and 1-iodo-3-methyl-phenyl is located in the threonyl-binding region (Figure 5).

According to biochemical testing, N-benzylidene- $N^{\prime}$-(4phenyl-thiazol-2-yl)-hydrazine derivatives possess inhibitory activity toward $S$. aureus ThrRS. It should be noted that earlier, the compounds from this chemical class were reported by us as the inhibitors of $M$. tuberculosis leucyl-tRNA synthetase and methionyl-tRNA synthetase. ${ }^{32,33}$ Therefore, the derivatives of $N$-benzylidene- $N^{\prime}$-(4-phenyl-thiazol-2-yl)-hydrazine inhibit both classes of aminoacyl-tRNA synthetases.

As it can be seen from Table 4, among the 13 tested $N$ benzylidene- $N^{\prime}$-(4-phenyl-thiazol-2-yl)-hydrazine derivatives, the most active compound -2,4-dibromo-6-\{[4-(4-nitrophenyl)-thiazol-2-yl]-hydrazonomethyl $\}$-phenol (compound 13) inhibits ThrRS by $72.65 \%$. The $\mathrm{IC}_{50}$ value for this

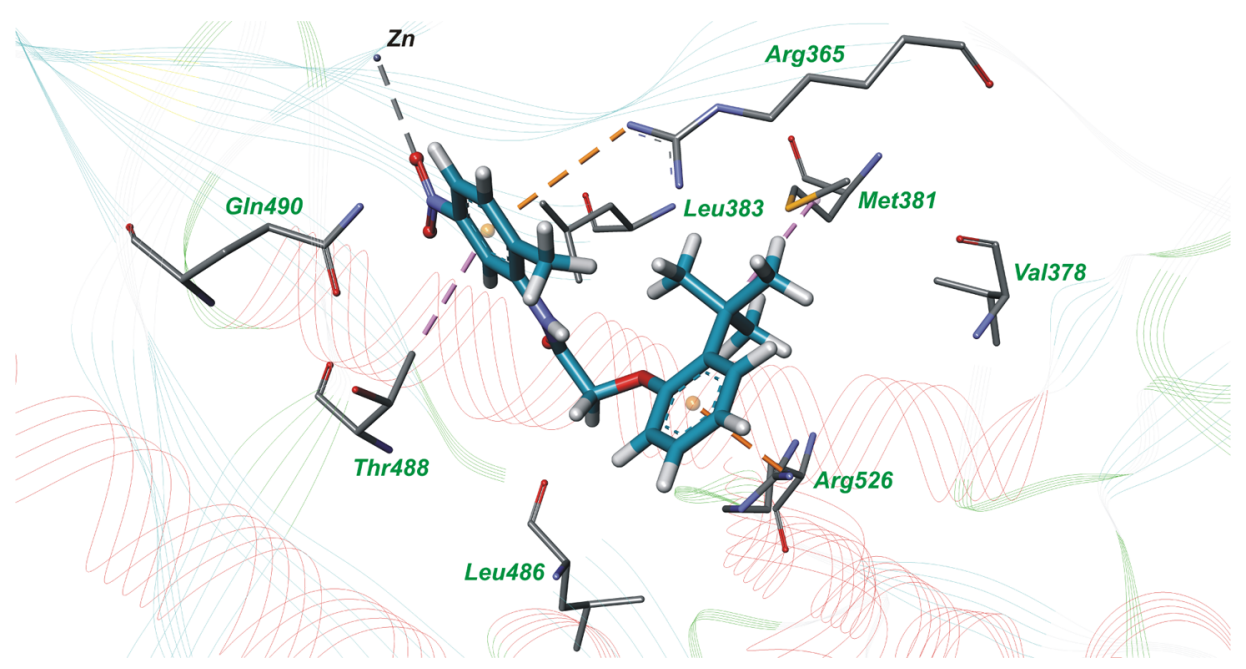

Figure 3. Complex of 2-(2-tert-butyl-phenoxy)- $\mathrm{N}$-(2-methyl-5-nitro-phenyl)-acetamide (compound 2) with amino acid residues in the active site of $S$. aureus ThrRS. The hydrophobic interactions are indicated with magenta dashed lines, $\pi$-cation interactions are shown by orange dashed lines, and the metal-acceptor bond is presented by the gray dashed line. 


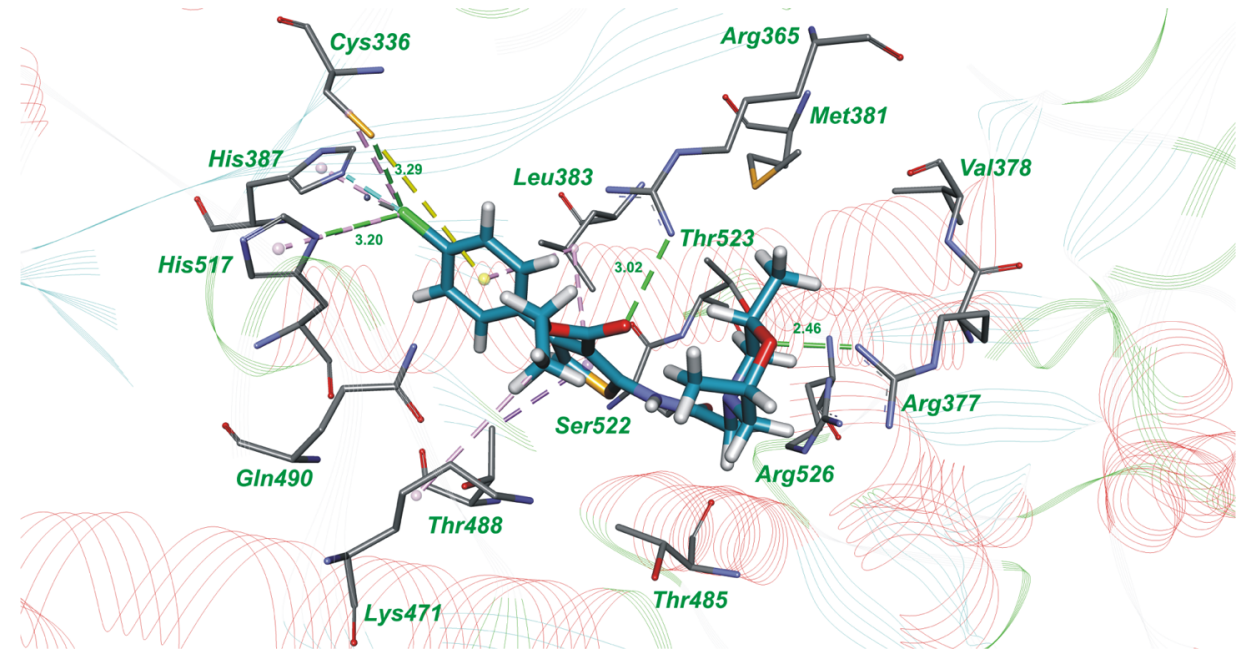

Figure 4. Complex of 4-(4-chloro-phenyl)-2-[2-(2,6-dimethyl-morpholin-4-yl)-acetylamino]-thiophene-3-carboxylic acid ethyl ester (compound 8) with amino acid residues in the active site of $S$. aureus ThrRS. The hydrogen bonds are shown by green dashed lines with the distances indicated in $\AA$, the hydrophobic interactions are indicated with magenta dashed lines, $\pi$-sulfur interaction is presented by yellow dashed lines, and the halogen bond is shown by blue dashed lines.

Table 3. Structures and In Vitro Inhibitory Activity of 1Phenyl-pyrrolidine-2,5-dione Derivatives toward $S$. aureus ThrRS

\begin{tabular}{|l|l|l|}
\hline No & $\begin{array}{c}\text { Residual activity } \\
\text { of ThrRs, \% }\end{array}$ \\
\hline 12 & \\
\hline 12
\end{tabular}

compound is $56.5 \pm 3.5 \mu \mathrm{M}$ (measured in duplicates). Molecular docking results demonstrate that nitro-phenyl interacts with the amino acid residues in the threonyl-binding region of ThrRS and 2,4-dibromophenyl is located in the adenine-binding region of $S$. aureus ThrRS (Figure 6).

We have tested five hit compounds for cytotoxicity toward the human cell line HEK293 using standard MTT assay. ${ }^{34}$ It was found that compounds 1, 2, 8, and 13 are not cytotoxic at a concentration of $100 \mu \mathrm{M}$ and only compound 11 reveals slight toxicity, decreasing the growth of HEK 293 cells by $30 \%$ at a concentration of $100 \mu \mathrm{M}\left(\mathrm{EC}_{50}>100 \mu \mathrm{M}\right)$.

\section{CONCLUSIONS}

Using the rational design approach, we identified five hit compounds from different chemical classes, inhibiting S. aureus threonyl-tRNA synthetase (ThrRS) by more than $70 \%$ at a concentration of $100 \mu \mathrm{M}$. The most active compound 2,4dibromo-6-\{[4-(4-nitro-phenyl)-thiazol-2-yl]-hydrazonomethyl $\}$-phenol has an $\mathrm{IC}_{50}$ value of $56.5 \pm 3.5 \mu \mathrm{M}$. According to the results of molecular docking, the inhibitors interact simultaneously with adenine- and threonyl-binding regions of $S$. aureus ThrRS. These compounds are not cytotoxic toward the human cell line HEK293 and can be used for further chemical optimization and biological research. It should be noted that the derivatives of $N$-benzylidene- $N^{\prime}$-(4-phenylthiazol-2-yl)-hydrazine inhibit both classes of aminoacyl-tRNA synthetases from different microorganisms and can be considered for the development of broad spectrum antibiotics.

\section{METHODS}

Molecular Docking. Semiflexible molecular docking of the small-molecular compounds into the active site of the $S$. aureus ThrRS crystal structure (PDB ID: 1NYQ) was performed with the program DOCK 4.0. ${ }^{35-38}$ The molecules of $5^{\prime}-\mathrm{O}-(\mathrm{N}-(\mathrm{L}-$ threonyl)-sulfamoyl)adenosine and water were removed from the crystal structure, but the $\mathrm{Zn}$ ion was kept in the active site.

The ligand geometry was calculated using the YFF force field. ${ }^{39}$ The partial atomic charges for the ligands were assigned with the Kirchhoff method. ${ }^{40}$ The partial atomic charges for the receptor were set with the Amber force field. The spheres of the active site were predicted using sphgen software from the DOCK package. The spheres which were outside of the aminoacyl-adenylate binding site of ThrRS were deleted manually. The energy grids of the receptor were generated using the grid program from the DOCK package.

The docking of the compound library into the aminoacyladenylate binding site of ThrRS was performed using the parameters described earlier. ${ }^{32}$

After the docking procedure, we have performed complex analysis to select compounds for in vitro screening. At first, compounds were ranged based on score values (less than -40 $\mathrm{kcal} / \mathrm{mol}$ ). Then, the compounds were evaluated for the ability to form hydrogen bonds with amino acid residues in the aminoacyl adenylate binding site of $S$. aureus ThrRS, such as Tyr468, Met334, Gln490, Asp385, Arg365, Ser522, Thr523, and Val378 using our in-house program y_hbonds, which is based on the analysis of distances and angles between the respective donor and acceptor atoms. Considering these criteria, we have chosen about 12,000 compounds for further visual inspection of receptor-ligand complexes. During visual 


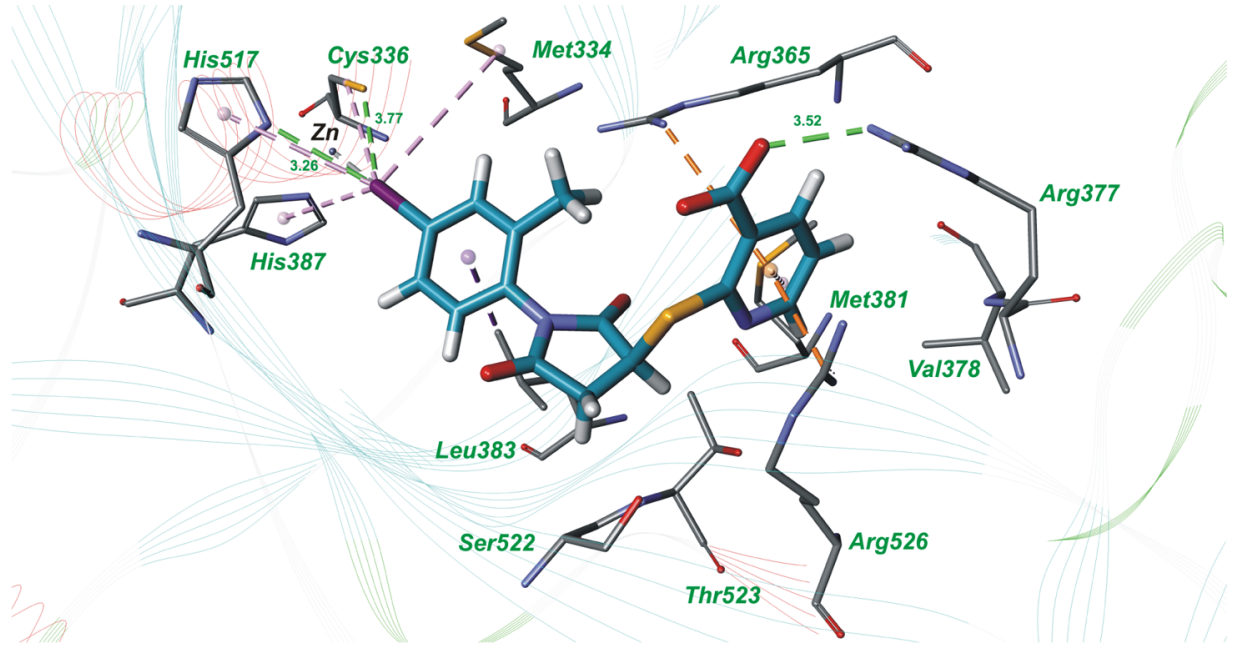

Figure 5. Complex of 2-[1-(4-iodo-2-methyl-phenyl)-2,5-dioxo-pyrrolidin-3-ylsulfanyl]-nicotinic acid (compound 11) with amino acid residues in the active site of $S$. aureus ThrRS. The hydrogen bonds are shown by green dashed lines with the distances indicated in $\AA$, the hydrophobic interactions are presented by magenta dashed lines, $\pi$-cation interactions are indicated with orange dashed lines, and the metal-acceptor bond is shown by gray dashed lines.

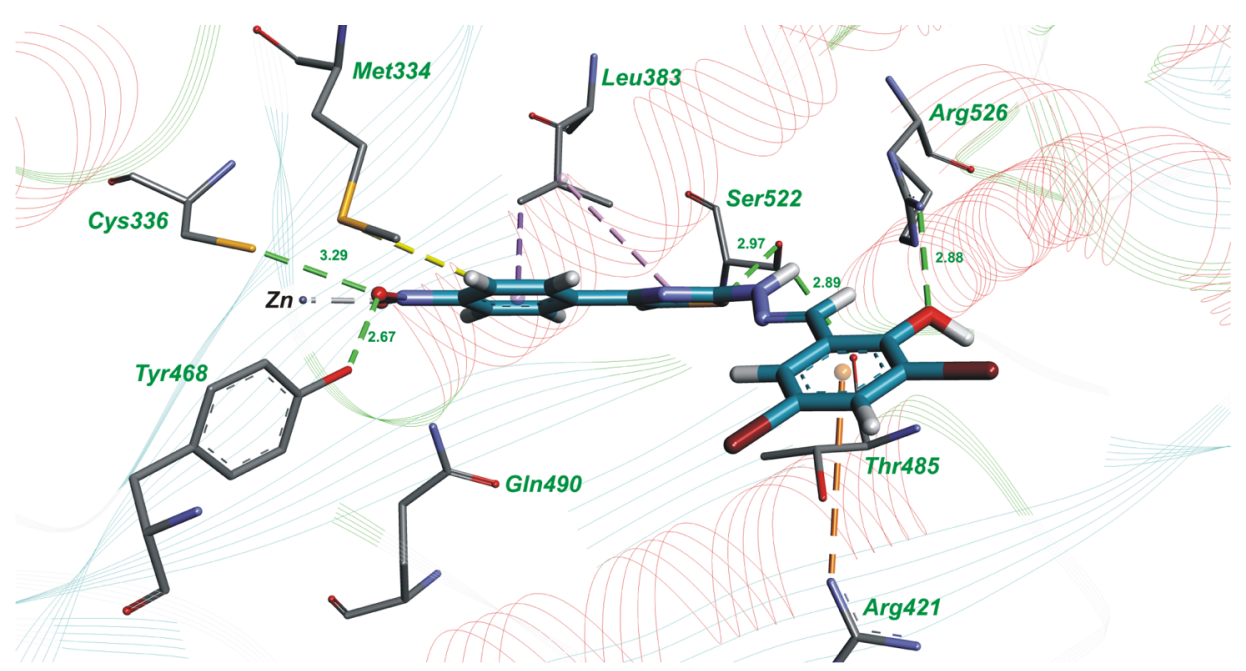

Figure 6. Complex of 2,4-dibromo-6-\{[4-(4-nitro-phenyl)-thiazol-2-yl]-hydrazonomethyl $\}$-phenol (compound 13) with amino acid residues in the active site of $S$. aureus ThrRS. The hydrogen bonds are shown by green dashed lines with the distances indicated in $\AA$, the hydrophobic interactions are presented by magenta dashed lines, $\pi$-cation interaction is indicated with orange dashed lines, $\pi$-sulfur interaction is presented by yellow dashed lines, and the metal-acceptor bond is shown by gray dashed lines.

analysis, we evaluated correctness of torsion angles, stacking interactions, complementarity of ligand and receptor surfaces, and so forth. Taking into account all these parameters, we have selected 180 compounds for investigation of their inhibitory activity toward recombinant $S$. aureus ThrRS.

Visual analysis of the receptor-ligand complexes was performed using Discovery Studio Visualizer 4.0. ${ }^{41}$

Cloning of ThrRS. The gene encoding ThrRS with restriction sites for $\mathrm{NcoI}$ and HindIII was synthesized by ATG/biosynthetics GmbH (Merzhausen, Germany) based on sequence information of the $S$. aureus ThrRS gene (GenBank ID: ABD30857.1). The linearized DNA fragment pGE(NcoIHindIII)-ThrRS-S was ligated to the plasmid vector pET28b (Novagen) which was previously dephosphorylated with phosphatase in $1 \times$ reaction buffer (Roche). Ligation was performed with T4 DNA ligase in $1 \times$ ligase buffer (Promega) for $2 \mathrm{~h}$ at $23{ }^{\circ} \mathrm{C}$. E. coli TOP10 cells (Invitrogen) were transformed by electroporation using a Bio-Rad Gene Pulsar.
The screening of positive clones was performed using the GeneJET Plasmid Miniprep Kit (Thermo Scientific). Positive clones were identified using restrictases NcoI and HindIII FastDigest (Thermo Scientific).

ThrRS Expression. The plasmid pET28b-ThrRS was transformed into E. coli BL21(DE3)pLysS and Lemo21(DE3) competent cells. The level of expression was analyzed in LB (Lauria-Broth), TB (Terrific-Broth), phosphate, and $2 \times Y$ T media with $50 \mu \mathrm{g} / \mathrm{mL}$ kanamycin. The bacterial growth was continued until the $\mathrm{OD}_{600}$ reached around 0.6. The protein synthesis was induced by IPTG in the range of concentration from $0.025 \mathrm{mM}$ to $1 \mathrm{mM}$ at 18,25 , and $37^{\circ} \mathrm{C}$.

Aliquots after 3-4 $\mathrm{h}$ and overnight induction were analyzed for solubility according to the protocol of Zerbs et al. ${ }^{42}$

For ThrRS expression, a modified ${ }^{43}$ autoinduction proto$\mathrm{col}^{44}$ was also used. E. coli BL21(DE3)pLysS and Lemo21(DE3) cells harboring the plasmid pET28b-ThrRS were incubated at $37{ }^{\circ} \mathrm{C}$ overnight in $2 \mathrm{~mL}$ of $2 \times \mathrm{YT}$ medium 
Table 4. Structures and In Vitro Inhibitory Activity of $\mathrm{N}$ Benzylidene- $N^{\prime}$-(4-phenyl-thiazol-2-yl)-hydrazine Derivatives toward $S$. aureus ThrRS

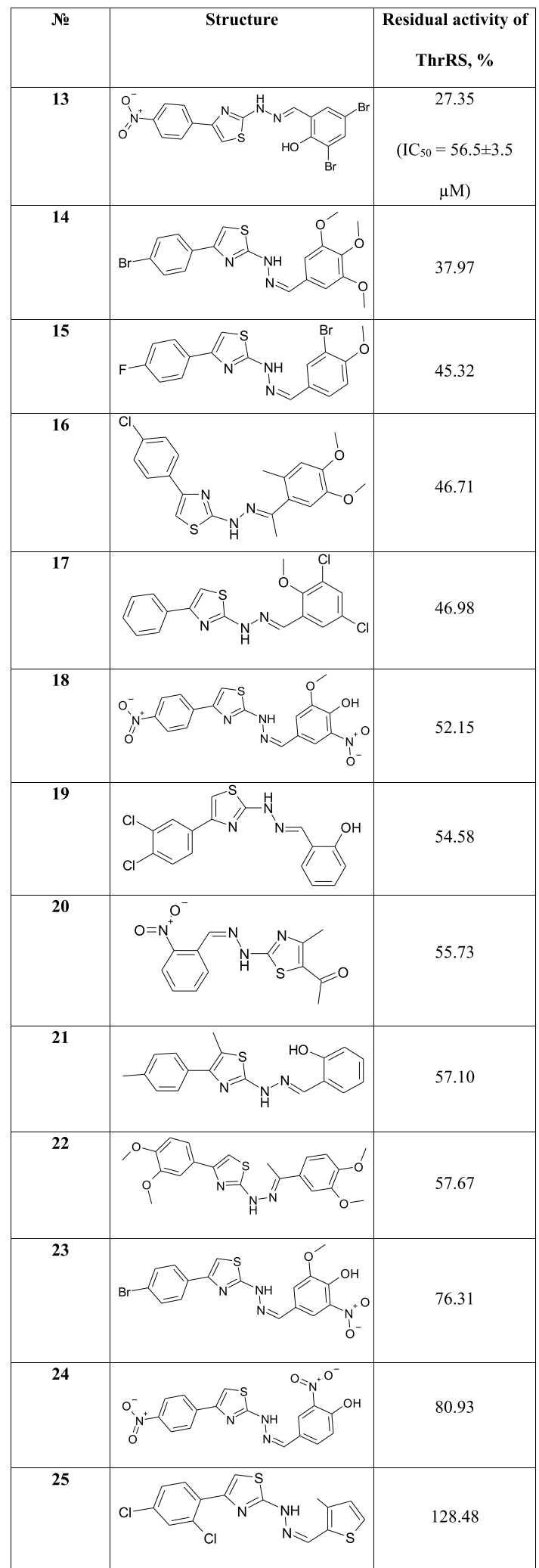

containing $50 \mu \mathrm{g} / \mathrm{mL}$ kanamycin and $1 \%$ glucose. 1:1000 dilutions of the overnight culture were taken for inoculation of $2 \times Y T$ medium supplemented with $40 \mu \mathrm{g} / \mathrm{mL}$ kanamycin, 25 $\mathrm{mM}\left(\mathrm{NH}_{4}\right)_{2} \mathrm{SO}_{4}, 50 \mathrm{mM} \mathrm{KH} \mathrm{PO}_{4}, 50 \mathrm{mM} \mathrm{Na} \mathrm{HPO}_{4}, 1 \mathrm{mM}$
$\mathrm{MgSO}_{4}, 0.05 \%$ glucose, $0.2 \% \alpha$-lactose, and $0.5 \%$ glycerol. The cultures were incubated at $18 / 30 / 37{ }^{\circ} \mathrm{C}$ for $16-18 \mathrm{~h}$ under conditions of intensive aeration.

Purification of S. aureus ThrRS. E. coli cells BL21(DE3)pLysS were grown in the medium $\mathrm{LB}$ until the $\mathrm{OD}_{600}$ reached 0.6 , and the expression of ThrRS was induced by $0.25 \mathrm{mM}$ IPTG for $3 \mathrm{~h}$ at $37{ }^{\circ} \mathrm{C}$. The cells were pelleted by centrifugation $\left(15 \mathrm{~min}\right.$ at $6000 \mathrm{~g}$ at $4{ }^{\circ} \mathrm{C}$ ), and the pellets were stored at $-80{ }^{\circ} \mathrm{C}$. The bacterial pellet was dissolved in buffer A containing $100 \mathrm{mM}$ Tris- $\mathrm{HCl}$ ( $\mathrm{pH} 8.0$ ), $300 \mathrm{mM}$ $\mathrm{NaCl}, 10 \mathrm{mM}$ DTT, $5 \mathrm{mM}$ PMSF, $4 \%$ glycerol, $1 \mathrm{mM} \mathrm{MgCl}_{2}$, $0.01 \mathrm{mg} / \mathrm{mL}$ lysozyme, and protease inhibitor cocktail tablet, EDTA-free. The cells were incubated on ice for $30 \mathrm{~min}$ and sonicated for $5 \mathrm{~min}$ with four sonicated bursts of $25 \mathrm{~s}$ followed by interval of $60 \mathrm{~s}$ for cooling. The cell pellet was precipitated by centrifugation at $20000 \mathrm{~g}$ for $25 \mathrm{~min}$ at $4{ }^{\circ} \mathrm{C}$. The supernatant was dialyzed in buffer B containing $20 \mathrm{mM}$ Tris$\mathrm{HCl}$ ( $\mathrm{pH} 8.0$ ), $2 \mathrm{mM}$ DTT, $0.1 \mathrm{mM} \mathrm{PMSF}$, and $5 \mathrm{mM} \mathrm{MgCl}$ for $3 \mathrm{~h}$ at $4{ }^{\circ} \mathrm{C}$. Then, the buffer was changed and dialyzed overnight. After dialysis, the lysate was loaded onto a DEAE Sepharose column (Amersham Biosciences) $(V=6.5 \mathrm{~mL})$, washed at a flow rate of $1 \mathrm{~mL} / \mathrm{min}$, and eluted with a gradient of $\mathrm{NaCl}$ from 25 to $500 \mathrm{mM}(2 \times 50 \mathrm{~mL})$. Fractions were analyzed by the Bradford method ${ }^{45}$ and SDS-PAGE. Fractions containing ThrRS were joined and concentrated on an Amicon Ultra-4 at $10 \mathrm{~K}$ MWCO spin column (Millipore, Billerica, MA) at $4{ }^{\circ} \mathrm{C}$ to remove salt impurities and loaded onto the Heparin Sepharose 6 Fast Flow column (GE Healthcare) $(0.5 \times 10$ $\mathrm{cm}$ ), washed with buffer $\mathrm{B}$ and eluted with a gradient of $\mathrm{KCl}$ from 0 to $300 \mathrm{mM}(2 \times 25 \mathrm{~mL})$. The peak fractions, containing ThrRS, were joined and concentrated on Amicon Ultra-4 (7000 g, $\left.4{ }^{\circ} \mathrm{C}\right)$.

Protein concentrations were determined by the Bradford method. ${ }^{45}$ The absorption coefficient at $\lambda=280 \mathrm{~nm}\left(\varepsilon_{280}=\right.$ $\left.73355 \mathrm{M}^{-1} \mathrm{~cm}^{-1}\right)$ and the absorption of $0.1 \%$ solution $\left(A_{280}(1\right.$ $\mathrm{mg} / \mathrm{mL})=0.985 \mathrm{mg}^{-1} \mathrm{~mL}$ ) were calculated from the $S$. aureus ThrRS amino acid sequence using the ExPASy ProtParam tool $^{46}$ and taken for determination of enzyme concentration.

In Vitro Aminoacylation Assay. The standard aminoacylation assay was performed in the reaction mixture $(20 \mu \mathrm{M})$ containing $50 \mathrm{mM}$ HEPES-NaOH (pH 7.5), $20 \mathrm{mM} \mathrm{MgCl}_{2}, 5$ $\mathrm{mM} \beta$-mercaptoethanol, $30 \mathrm{mM} \mathrm{KCl}, 100 \mu \mathrm{g} / \mathrm{mL} \mathrm{BSA}, 4 \mathrm{mg} /$ $\mathrm{mL}$ of the total E. coli MRE600 tRNA, $27 \mu \mathrm{M}\left[{ }^{14} \mathrm{C}\right]-\mathrm{L}-\mathrm{Thr}$, and $500 \mathrm{nM}$ recombinant $S$. aureus ThrRS with appropriate concentrations of the compound (dissolved in DMSO). The reactions were initiated by the addition of $10 \mathrm{mM}$ ATP and incubated for $10 \mathrm{~min}$ at $37{ }^{\circ} \mathrm{C}$. The reaction was stopped by the addition of $10 \%$ trichloroacetic acid and was loaded on the GF/C filter, washed with 5\% trichloroacetic acid, dried, and counted using a scintillation counter. Aliquots were quenched with $10 \%$ trichloracetic acid, and the level of tRNA aminoacylation was measured using a scintillation counter [Hidex 600 SL liquid scintillation analyzer (Finland)].

MTT Assay. The toxicity of compounds toward the HEK293 cell was examined using a standard MTT assay. ${ }^{35}$ The cells were cultivated in Dulbecco's modified Eagle's medium supplemented with $10 \%$ fetal bovine serum, $100 \mathrm{mg} /$ $\mathrm{mL}$ streptomycin, and $100 \mathrm{mg} / \mathrm{mL}$ penicillin in humidified air with $5 \% \mathrm{CO}_{2}$ at $37{ }^{\circ} \mathrm{C}$. The cells were seeded into 96-well plates at a concentration of $2 \times 10^{5}$ cells $/ \mathrm{mL}$ and grown for 24 h. Then, the cells were supplemented with compounds in DMSO solution (final DMSO concentration was less than $0.5 \%)$ at different concentrations. After $72 \mathrm{~h}$ of incubation, the 
cells were treated with $15 \mu \mathrm{L}$ of MTT solution $(5 \mathrm{mg} / \mathrm{mL})$ for $4 \mathrm{~h}$ at $37{ }^{\circ} \mathrm{C}, 5 \% \mathrm{CO}_{2}$. The formazan precipitates were dissolved in $200 \mu \mathrm{L}$ of DMSO, and the absorbance at $\lambda=540$ $\mathrm{nm}$ was measured with spectrofluorometer MR 700 (Dynatech). The cell viability was calculated as a percentage relative to intact control cells.

\section{ASSOCIATED CONTENT}

\section{SI Supporting Information}

The Supporting Information is available free of charge at https://pubs.acs.org/doi/10.1021/acsomega.1c03789.

Analysis of ThrRS expression in E. coli BL21(DE3)pLysS cells; analysis of expression of the ThrRS soluble fraction with induction by 0.5 or $1 \mathrm{mM}$ IPTG concentrations at $37{ }^{\circ} \mathrm{C}$; analysis of expression of the ThrRS soluble fraction with induction by $0.25,0.5$, or 1 $\mathrm{mM}$ IPTG concentrations at $25^{\circ} \mathrm{C}$; analysis of ThrRS expression at 0.1 and $0.25 \mathrm{mM}$ IPTG concentration and in the medium with $3 \%$ ethanol after induction for $3 \mathrm{~h}$ at $37{ }^{\circ} \mathrm{C}$; expression of the ThrRS soluble fraction with induction by $0.1,0.25$, and $1 \mathrm{mM}$ IPTG concentration and in the medium with $3 \%$ ethanol for $3 \mathrm{~h}$ at 18 and 25 ${ }^{\circ} \mathrm{C}$; expression of the ThrRS soluble fraction after overnight induction at 18 and $25{ }^{\circ} \mathrm{C}$; expression of the ThrRS soluble fraction in Lemo21(DE3) E. coli cells in $\mathrm{LB}$ medium after $4 \mathrm{~h}$ of induction at $30^{\circ} \mathrm{C}$ with $0.4 \mathrm{mM}$ IPTG and various concentrations of L-rhamnose; expression of the ThrRS soluble fraction in Lemo21(DE3) E. coli cells in LB medium after overnight induction at $30{ }^{\circ} \mathrm{C}$; expression of $S$. aureus ThrRS using the autoinduction protocol; SDS-PAGE analysis of soluble and insoluble fractions of E. coli proteins after induction expression of $S$. aureus ThrRS by autoinduction conditions $(10 \mu \mathrm{L}$ of culture per lane was loaded); elution profile (absorbance at $280 \mathrm{~nm}$ versus elution volume) of the anion exchanger DEAESepharose; purification of $S$. aureus ThrRS using the DEAE-Sepharose column; purification of $S$. aureus ThrRS using the heparin-Sepharose column; superposition of compound $\mathbf{1}$ (carbon atoms are labeled with blue color) and compound $\mathbf{2}$ (carbon atoms are labeled with magenta color) in the active site of $S$. aureus threonyl-tRNA synthetase; and structures and in vitro inhibitory activity toward $S$. aureus ThrRS for compounds, selected according to molecular docking results (PDF)

\section{AUTHOR INFORMATION}

\section{Corresponding Author}

Galyna P. Volynets - Department of Medicinal Chemistry, Institute of Molecular Biology and Genetics National Academy of Sciences of Ukraine, Kyiv 03143, Ukraine; The Scientific-Services Company "OTAVA", Kyiv 03143, Ukraine; ○ orcid.org/0000-0002-0166-2642; Email: g.p.volynets@gmail.com

\section{Authors}

Mariia Yu. Rybak - Department of Protein Synthesis Enzymology, Institute of Molecular Biology and Genetics National Academy of Sciences of Ukraine, Kyiv 03143, Ukraine; ○ orcid.org/0000-0002-0478-0222
Olga I. Gudzera - Department of Protein Synthesis Enzymology, Institute of Molecular Biology and Genetics National Academy of Sciences of Ukraine, Kyiv 03143, Ukraine

Oksana B. Gorbatiuk - Department of Cell Regulatory Mechanisms, Institute of Molecular Biology and Genetics National Academy of Sciences of Ukraine, Kyiv 03143, Ukraine

Mariia O. Usenko - Department of Cell Regulatory Mechanisms, Institute of Molecular Biology and Genetics National Academy of Sciences of Ukraine, Kyiv 03143, Ukraine

Sergiy M. Yarmoluk - Department of Medicinal Chemistry, Institute of Molecular Biology and Genetics National Academy of Sciences of Ukraine, Kyiv 03143, Ukraine

Michael A. Tukalo - Department of Protein Synthesis Enzymology, Institute of Molecular Biology and Genetics National Academy of Sciences of Ukraine, Kyiv 03143, Ukraine

Complete contact information is available at:

https://pubs.acs.org/10.1021/acsomega.1c03789

\section{Notes}

The authors declare no competing financial interest.

\section{ACKNOWLEDGMENTS}

This work was supported by the NAS of the Ukraine grant for young scientists (project no 0120U000079) for 2020-2021.

\section{REFERENCES}

(1) Krishna, S.; Miller, L. S. Host-pathogen interactions between the skin and Staphylococcus aureus. Curr. Opin. Microbiol. 2012, 15, 2835.

(2) Welte, T.; Kantecki, M.; Stone, G. G.; Hammond, J. Ceftaroline fosamil as a potential treatment option for Staphylococcus aureus community-acquired pneumonia in adults. Int. J. Antimicrob. Agents 2019, 54, 410-422.

(3) Jang, Y.-R.; Kim, T.; Kim, M.-C.; Sup Sung, H.; Kim, M.-N.; Kim, M. J.; Kim, S. H.; Lee, S.-O.; Choi, S.-H.; Woo, J. H.; Kim, Y. S.; Chong, Y. P. Sternoclavicular septic arthritis caused by Staphylococcus aureus: excellent results from medical treatment and limited surgery. Infect. Dis. 2019, 51, 694-700.

(4) Tascini, C.; Attanasio, V.; Ripa, M.; Carozza, A.; Pallotto, C.; Bernardo, M.; Francisci, D.; Oltolini, C.; Palmiero, G.; Scarpellini, P. Ceftobiprole for the treatment of infective endocarditis: a case series. J. Glob. Antimicrob. Resist. 2020, 20, 56-59.

(5) Dugourd, P.-M.; Dupont, A.; Hubiche, T.; Chiaverini, C.; Alkhalifa, A.; Roudiere, L.; Tristan, A.; Gustave, C.-A.; Del Giudice, P. Érythème généralisé fébrile et choc : choc toxinique staphylococcique. Ann. Dermatol. Venereol. 2019, 146, 287-291.

(6) Toledo, A. G.; Golden, G.; Campos, A. R.; Cuello, H.; Sorrentino, J.; Lewis, N.; Varki, N.; Nizet, V.; Smith, J. W.; Esko, J. D. Proteomic atlas of organ vasculopathies triggered by Staphylococcus aureus sepsis. Nat. Commun. 2019, 10, 4656.

(7) Bergin, S. P.; Holland, T. L.; Fowler, V. G., Jr.; Tong, S. Y. C. Bacteremia, sepsis, and infective endocarditis associated with Staphylococcus aureus. Curr. Top. Microbiol. Immunol. 2015, 409, 263-296.

(8) Zhang, Y.; Zhang, J.; Chen, W.; Angsantikul, P.; Spiekermann, K. A.; Fang, R. H.; Gao, W.; Zhang, L. Erythrocyte membrane-coated nanogel for combinatorial antivirulence and responsive antimicrobial delivery against Staphylococcus aureus infection. J. Controlled Release 2017, 263, 185-191.

(9) Gardete, S.; Tomasz, A. Mechanisms of vancomycin resistance in Staphylococcus aureus. J. Clin. Invest. 2014, 124, 2836-2840. 
(10) Kali, A. Antibiotics and bioactive natural products in treatment of methicillin resistant Staphylococcus aureus: A brief review. Pharmacogn. Rev. 2015, 9, 29-34.

(11) Kaur, D.; Chate, S. Study of antibiotic resistance pattern in methicillin resistant staphylococcus aureus with special reference to newer antibiotic. J. Global Infect. Dis. 2015, 7, 78-84.

(12) Arunkumar, V.; Prabagaravarthanan, R.; Bhaskar, M. Prevalence of methicillin-resistant Staphylococcus aureus (MRSA) infections among patients admitted in critical care units in a tertiary care hospital. Int. J. Res. Med. Sci. 2017, 5, 2362-2366.

(13) McGuinness, W. A.; Malachowa, N.; DeLeo, F. R. Vancomycin Resistance in Staphylococcus aureus. Yale J. Biol. Med. 2017, 90, 269281.

(14) Marty, F. M.; Yeh, W. W.; Wennersten, C. B.; Venkataraman, L.; Albano, E.; Alyea, E. P.; Gold, H. S.; Baden, L. R.; Pillai, S. K. Emergence of a clinical daptomycin-resistant Staphylococcus aureus isolate during treatment of methicillin-resistant Staphylococcus aureus bacteremia and osteomyelitis. J. Clin. Microbiol. 2006, 44, 595-597.

(15) Ikeda-Dantsuji, Y.; Hanaki, H.; Sakai, F.; Yanagisawa, C.; Nakae, T.; Sakai, F.; Tomono, K.; Takesue, Y.; Honda, J.; Nonomiya, Y.; Suwabe, A.; Nagura, O.; Yanagihara, K.; Mikamo, H.; Fukuchi, K.; Kaku, M.; Kohno, S.; Yoshida, K.; Niki, Y. Linezolid-resistant Staphylococcus aureus isolated from 2006 through 2008 at six hospitals in Japan. J. Infect. Chemother. 2011, 17, 45-51.

(16) Pang, L.; Weeks, S. D.; Van Aerschot, A. Aminoacyl-tRNA synthetases as valuable targets for antimicrobial drug discovery. Int. J. Mol. Sci. 2021, 22, 1750.

(17) Kwon, N. H.; Fox, P. L.; Kim, S. Aminoacyl-tRNA synthetases as therapeutic targets. Nat. Rev. Drug Discovery 2019, 18, 629-650.

(18) Ho, J. M.; Bakkalbasi, E.; Söll, D.; Miller, C. A. Drugging tRNA aminoacylation. RNA Biol. 2018, 15, 667-677.

(19) Ribas de Pouplana, L.; Schimmel, P. Two classes of tRNA synthetases suggested by sterically compatible dockings on tRNA acceptor stem. Cell 2001, 104, 191-193.

(20) Eriani, G.; Delarue, M.; Poch, O.; Gangloff, J.; Moras, D. Partition of tRNA synthetases into two classes based on mutually exclusive sets of sequence motifs. Nature 1990, 347, 203-206.

(21) Novoa, E. M.; Camacho, N.; Tor, A.; Wilkinson, B.; Moss, S.; Marín-García, P.; Azcárate, I. G.; Bautista, J. M.; Mirando, A. C.; Francklyn, C. S.; Varon, S.; Royo, M.; Cortés, A.; Ribas de Pouplana, L. Analogs of natural aminoacyl-tRNA synthetase inhibitors clear malaria in vivo. Proc. Natl. Acad. Sci. U.S.A. 2014, 111, E5508-E5517. (22) Saint-Léger, A.; Sinadinos, C.; Ribas de Pouplana, L. The growing pipeline of natural aminoacyl-tRNA synthetase inhibitors for malaria treatment. Bioengineered 2016, 7, 60-64.

(23) Hütter, R.; Poralla, K.; Zachau, H. G.; Zähner, H. Metabolic products of microorganisms. 5l. On the mechanism of action of borrelidin-inhibition of the threonine incorporation in sRNA. Biochem. Z. 1966, 344, 190-196.

(24) Paetz, W.; Nass, G. Biochemical and Immunological Characterization of Threonyl-tRNA Synthetase of Two Borrelidin-Resistant Mutants of Escherichia coli K12. Eur. J. Med. Chem. 1973, 35, 331337.

(25) Wilkinson, B.; Gregory, M. A.; Moss, S. J.; Carletti, I.; Sheridan, R. M.; Kaja, A.; Ward, M.; Olano, C.; Mendez, C.; Salas, J. A.; Leadlay, P. F.; van Ginckel, R.; Zhang, M.-Q. Separation of antiangiogenic and cytotoxic activities of borrelidin by modification at the C17 side chain. Bioorg. Med. Chem. Lett. 2006, 16, 5814-5817.

(26) Sugawara, A.; Tanaka, T.; Hirose, T.; Ishiyama, A.; Iwatsuki, M.; Takahashi, Y.; Otoguro, K.; Ōmura, S.; Sunazuka, T. Borrelidin analogues with antimalarial activity: design, synthesis and biological evaluation against Plasmodium falciparum parasites. Bioorg. Med. Chem. Lett. 2013, 23, 2302-2305.

(27) Teng, M.; Hilgers, M. T.; Cunningham, M. L.; Borchardt, A.; Locke, J. B.; Abraham, S.; Haley, G.; Kwan, B. P.; Hall, C.; Hough, G. W.; Shaw, K. J.; Finn, J. Identification of bacteria-selective threonyltRNA synthetase substrate inhibitors by structure-based design. J. Med. Chem. 2013, 56, 1748-1760.
(28) Guo, J.; Chen, B.; Yu, Y.; Cheng, B.; Cheng, Y.; Ju, Y.; Gu, Q.; $\mathrm{Xu}, \mathrm{J}$.; Zhou, H. Discovery of novel tRNA-amino acid dual-site inhibitors against threonyl-tRNA synthetase by fragment-based target hopping. Eur. J. Med. Chem. 2020, 187, 111941.

(29) Guo, J.; Chen, B.; Yu, Y.; Cheng, B.; Ju, Y.; Tang, J.; Cai, Z.; $\mathrm{Gu}$, Q.; Xu, J.; Zhou, H. Structure-guided optimization and mechanistic study of a class of quinazolinone-threonine hybrids as antibacterial ThrRS inhibitors. Eur. J. Med. Chem. 2020, 207, 112848.

(30) Torres-Larios, A.; Sankaranarayanan, R.; Rees, B.; DockBregeon, A.-C.; Moras, D. Conformational movements and cooperativity upon amino acid, ATP and tRNA binding in threonyltRNA synthetase. J. Mol. Biol. 2003, 331, 201-211.

(31) We used a database of commercially available compounds; Otava Ltd., http://www.otavachemicals.com, (accessed April, 2020).

(32) Kovalenko, O. P.; Volynets, G. P.; Rybak, M. Y.; Starosyla, S. A.; Gudzera, O. I.; Lukashov, S. S.; Bdzhola, V. G.; Yarmoluk, S. M.; Boshoff, H. I.; Tukalo, M. A. Dual-target inhibitors of mycobacterial aminoacyl-tRNA synthetases among N-benzylidene- $\mathrm{N}^{\prime}$-thiazol-2-ylhydrazines. Medchemcomm 2019, 10, 2161-2169.

(33) Gudzera, O. I.; Golub, A. G.; Bdzhola, V. G.; Volynets, G. P.; Lukashov, S. S.; Kovalenko, O. P.; Kriklivyi, I. A.; Yaremchuk, A. D.; Starosyla, S. A.; Yarmoluk, S. M.; Tukalo, M. A. Discovery of potent anti-tuberculosis agents targeting leucyl-tRNA synthetase. Bioorg. Med. Chem. 2016, 24, 1023-1031.

(34) Mosmann, T. Rapid colorimetric assay for cellular growth and survival: application to proliferation and cytotoxicity assays. J. Immunol. Methods 1983, 65, 55-63.

(35) Bodian, D. L.; Yamasaki, R. B.; Buswell, R. L.; Stearns, J. F.; White, J. M.; Kuntz, I. D. Inhibition of the fusion-inducing conformational change of influenza hemagglutinin by benzoquinones and hydroquinones. Biochemistry 1993, 32, 2967-2978.

(36) Ewing, T. J. A.; Makino, S.; Skillman, A. G.; Kuntz, I. D. DOCK 4.0: search strategies for automated molecular docking of flexible molecule databases. J. Comput.-Aided Mol. Des. 2001, 15, 411-428.

(37) Ring, C. S.; Sun, E.; McKerrow, J. H.; Lee, G. K.; Rosenthal, P. J.; Kuntz, I. D.; Cohen, F. E. Structure-based inhibitor design by using protein models for the development of antiparasitic agents. Proc. Natl. Acad. Sci. U.S.A. 1993, 90, 3583-3587.

(38) Shoichet, B. K.; Stroud, R. M.; Santi, D. V.; Kuntz, I. D.; Perry, K. M. Structure-based discovery of inhibitors of thymidylate synthase. Science 1993, 259, 1445-1450.

(39) Yakovenko, O. Y.; Oliferenko, A.; Golub, A.; Bdzhola, V.; Yarmoluk, $S$. The new method of distribution integrals evaluations for high throughput virtual screening. Ukr. Bioorg. Acta 2007, 1, 52-62.

(40) Yakovenko, O.; Oliferenko, A. A.; Bdzhola, V. G.; Palyulin, V. A.; Zefirov, N. S. Kirchhoff atomic charges fitted to multipole moments: Implementation for a virtual screening system. J. Comput. Chem. 2008, 29, 1332-1343.

(41) Discovery Studio Visualizer 4.0, 2019. https://www.3dsbiovia. $\mathrm{com} /$ products/collaborative-science/biovia-discovery-studio/ visualization-download.php, (accessed May, 2019).

(42) Zerbs, S.; Frank, A. M.; Collart, F. R. Chapter 12 Bacterial Systems for Production of Heterologous Proteins. Methods Enzymol. 2009, 463, 149-168.

(43) Gorbatiuk, O. B.; Tsapenko, M. V.; Pavlova, M. V.; Okunev, O. V.; Kordium, V. A. Bioaffinity sorbent based on immobilized protein A Staphylococcus aureus: development and application. Biopolym. Cell 2012, 28, 141-148.

(44) Studier, F. W. Protein production by auto-induction in highdensity shaking cultures. Protein Expression Purif. 2005, 41, 207-234.

(45) Bradford, M. M. A rapid and sensitive method for the quantitation of microgram quantities of protein utilizing the principle of protein-dye binding. Anal. Biochem. 1976, 72, 248-254.

(46) Gasteiger, E.; Gattiker, A.; Hoogland, C.; Ivanyi, I.; Appel, R. D.; Bairoch, A. ExPASy: The proteomics server for in-depth protein knowledge and analysis. Nucleic Acids Res. 2003, 31, 3784-3788. 